\title{
Hidradenitis suppurativa and rheumatoid arthritis: evaluating the bidirectional association
}

\author{
Khalaf Kridin ${ }^{1,2,3}$ (1) Eran Shavit ${ }^{3,4} \cdot$ Giovanni Damiani $^{5,6} \cdot$ Arnon D Cohen $^{7,8,9}$
}

Received: 5 June 2021 / Accepted: 24 July 2021 / Published online: 19 August 2021

(c) The Author(s) 2021

\begin{abstract}
Despite some common pathogenic themes, the association of hidradenitis suppurativa (HS) and rheumatoid arthritis (RA) has been poorly investigated. We aimed to evaluate the bidirectional association between HS and RA. A population-based study was conducted to compare HS patients $(n=6779)$ with age-, sex- and ethnicity-matched control subjects $(n=33,260)$ with regard to the incidence of new-onset and the prevalence of preexisting RA. Adjusted hazard ratios (HRs) and adjusted odds ratios (ORs) were estimated. The prevalence of preexisting RA was greater among patients with HS relative to controls $(0.5 \%$ vs $0.3 \%$. respectively; $p=0.019)$. The odds of being diagnosed with HS were 1.6 -fold higher in patients with a history of RA (fully-adjusted OR, 1.66; 95\% CI, 1.11-2.49; $p=0.014$ ). The incidence rate of new-onset RA was estimated at 4.3 (95\% CI, 2.5-6.8) and 2.4 (95\% CI, 1.8-3.2) cases per 10,000 person-years among patients with HS and controls, respectively. The risk of RA was comparable between patients with HS and controls (fully-adjusted HR, 1.45; 95\% CI, 0.77-2.72; $p=0.249$ ). Compared to other patients with HS, those with HS and comorbid RA were older, had a higher prevalence of diabetes mellitus, hypertension, and hyperlipidemia, and had a comparable risk of all-cause mortality. In conclusions, a preexisting diagnosis of RA predisposes individuals to develop HS. Clinicians managing patients with HS and RA should be aware of this association. Further research is required to delineate the underlying pathomechanism of this observation.
\end{abstract}

Keywords Hidradenitis suppurativa $\cdot$ Rheumatoid arthritis $\cdot$ Risk $\cdot$ Association

Khalaf Kridin

dr_kridin@hotmail.com

1 Lübeck Institute of Experimental Dermatology, University of Lübeck, Ratzeburger Allee 160, 23562 Lübeck, Germany

2 Azrieli Faculty of Medicine, Bar-Ilan University, Safed, Israel

3 Unit of Dermatology and Skin Research Laboratory, Baruch Padeh Medical Center, Poriya, Israel

4 Dermatology Unit, Barzilai University Medical Center, Ashkelon, Israel

5 The Faculty of Health Sciences, Ben-Gurion University of the Negev, Beer-Sheba, Israel

6 Clinical Dermatology, IRCCS Istituto Ortopedico Galeazzi, Milan, Italy

7 Department of Biomedical, Surgical and Dental Sciences, University of Milan, Milan, Italy

8 Clalit Health Services, Tel-Aviv, Israel

9 Siaal Research Center for Family Medicine and Primary Care, Faculty of Health Sciences, Ben-Gurion University of the Negev, Beer Sheva, Israel

\section{Introduction}

Hidradenitis suppurativa (HS), previously termed 'acne inversa', is a chronic recurrent inflammatory cutaneous disease involving the pilosebaceous unit [1]. HS is characterized clinically by deep-seated nodules, abscesses, and draining sinus tracts that eventually end up as scaring tissue. These are located mostly in the groin, genital areas, and axillae, although the inframammary area, neck, and other anatomical regions may also be affected [2]. The prevalence of HS ranges between $0.05 \%$ and $4.0 \%$ in different study populations, and it is more frequent in women and smokers $[2,3]$. HS typically manifests in the second and third decades of life, and its incidence drops in postmenopausal women, signifying a hormonal influence on the pathogenesis of the disease $[4,5]$.

Rheumatoid arthritis (RA) is a chronic debilitating disease that manifests as persistent synovitis, which might lead to the destruction of joints and deformation of bones. It affects $0.5-1.0 \%$ of western populations, with the prototypical patient being a middle-aged woman. RA has a severe 
impact on patients' quality of life owing to its debilitating nature and chronic course [6].

Numerous anecdotal case reports suggested the coexistence of HS and RA in individual patients [7, 8]. However, the epidemiological association between these conditions is yet to be firmly established. In this study, we sought to evaluate the bidirectional association between HS and RA utilizing a large-scale population-based study. We additionally aimed to profile patients with HS and comorbid RA relative to HS patients without RA.

\section{Methods}

\section{Study design and database}

Aiming at elucidating the bidirectional association between HS and RA, the current study followed two study designs. A case-control study that evaluates the prevalence of preexisting RA among patients with HS was conducted to identify the odds of RA in individuals with a history of HS. In addition, a retrospective cohort design that longitudinally follows patients with HS for the incidence of new-onset RA was performed to assess the risk of RA after HS. The study was approved by the institutional review board (IRB) of BenGurion University in accordance with the declaration of Helsinki (approval code: 0212-17-COM).

The current study relied on data retrieved from the computerized database of Clalit Health Services (CHS). CHS is the largest healthcare maintenance organization in Israel, providing a wide assortment of private and public healthcare services for 4,540,768 enrollees as of October 2018, representing $52 \%$ of the general Israeli population. The computerized dataset of CHS continuously retrieves data from several sources covering both ambulatory and hospitalized care settings. CHS is additionally typified by free access to healthcare service, comprehensive documentation, and negligible loss to follow-up, which renders its database highly compatible for valid and reliable epidemiological data (8).

\section{Study population and definition of main variables and covariates}

To enroll the current study population, the computerized database of CHS was systematically screened for enrollees with a new-onset diagnosis of HS between January 2000 and December 2018. Patients were subject to inclusion if an (i) HS-specific code was registered by a CHS board-certified dermatologist or (ii) a diagnosis of HS was registered in discharge letters of patients admitted to dermatological inpatient wards $[9,10]$.

A control group encompassing up to 5 individuals per case of HS was additionally formed. Controls were matched to cases upon sex, age, and ethnicity. Control individuals were enrolled at the same date on which the diagnosis of HS was documented in the corresponding cases. The diagnosis of RA was based on its documentation in the chronic registry of CHS. Cases of RA were defined on the grounds of documentation by board-certified rheumatologists, the purchase of RA-related drugs, and suggestive laboratory and imaging data $[11,12]$.

Outcome measures were controlled for underlying comorbidities utilizing the Charlson comorbidity index (CCI), an epidemiological scale evaluating the severity of comorbidities among study participants. The latter was proved reliable in predicting mortality (9) and is under widespread usage in epidemiological studies. To avoid overadjustment bias, a modified version of CCI excluding collagen tissue diseases was adopted in the current analysis. Outcome measures were additionally adjusted for body mass index (BMI) and demographic variables. The date of death of study participants was ascertained by linking the study cohort with the National Registry of Deaths Database. In the mortality analysis, all eligible patients were followed up from the onset of HS until December 31st, 2018 , or death, whichever occurs earlier.

\section{Statistical analysis}

Baseline characteristics were described by means and standard deviations (SD)s for continuous variables and percentages for categorical variables. The comparison between subgroups was performed using the chi-square test and $t$-test for categorical and continuous variables, respectively.

Logistic regression was used to calculate odds ratios (ORs) and 95\% confidence intervals (CIs) to compare cases and control with respect to the presence of preexisting RA. The association was calculated based on individuals who developed HS following the diagnosis of RA in accordance with the presence of a temporal relationship between exposure and outcome in case-control studies.

Incidence rates of RA were calculated for both HS patients and controls and expressed as the number of events per 10,000 person-years. The incidence of these outcomes was calculated merely for individuals without a history of RA prior to the study initiation. Hazard ratios (HRs) and 95\% CIs for the risk of new-onset RA were obtained by the use of Cox regression models. The cumulative survival of HS patients with and without RA was calculated using Kaplan-Meier method and compared between the subgroups via stratified log-rank test. Two-tailed $p$-values less than 0.05 were considered statistically significant. All statistical analyses were performed using SPSS software, version 25 (SPSS, Armonk, NY: IBM Corp). 


\section{Results}

\section{Characteristics of the study population}

The current study was comprised of 6779 patients with HS and 33,260 age-, sex-, and ethnicity-matched control subjects. The mean (SD) age at the diagnosis of HS was 33.1 (15.1) years, and $4071(60.1 \%)$ were females. The mean BMI and the prevalence of smoking, diabetes mellitus, hyperlipidemia, and hypertension were significantly greater in cases than in controls. The demographic and clinical features of the study participants are outlined in Table 1 .

\section{The odds of hidradenitis suppurativa after rheumatoid arthritis (case-control design)}

The prevalence of preexisting RA was greater among patients with HS relative to controls $(0.5 \%$ vs $0.3 \%$. respectively, $p=0.019)$. Therefore, the odds of being diagnosed with HS were 1.6-fold higher in patients with a history of RA (OR, 1.59; 95\% CI, 1.07-2.36). In an age-, sex-, and, ethnicity-stratified analysis, the association of RA with subsequent HS was greater among individuals older than 40 years (OR, $1.73 ; 95 \% \mathrm{CI}, 1.08-2.79 ; p=0.022)$, males (OR, 2.22; 95\% CI, 1.01-4.89; $p=0.041$ ), and Arabs (OR, 2.93; 95\% CI, 1.15-7.45; $p=0.018$; Table 2). The association between a history of RA and a later diagnosis of HS was only significant in individuals in whom the diagnosis of RA preceded that of HS by more than 10 years (OR, 1.99; 95\% CI, 1.09-3.63; $p=0.022$; Table 2).

We then carried out a multivariate analysis adjusting for putative confounders. The association was robust to a model adjusting for age and sex (age- and sex-adjusted OR, 1.61; 95\% CI, 1.08-2.38; $p=0.019$ ) as well as to a model adjusting for age, sex, ethnicity, BMI, and comorbidities (fully adjusted OR, 1.66; 95\% CI, 1.11-2.49; $p=0.014$ ).

\section{The risk of rheumatoid arthritis in patients with hidradenitis suppurativa (retrospective cohort design)}

Patients with HS and controls were cumulatively followed for 37,591.7and 184,763.3 person-years, respectively. During this follow-up duration, the incidence rate of RA was estimated at 4.3 (95\% CI, 2.5-6.8) and 2.4 (95\% CI, 1.8-3.2) cases per 10,000 person-years in patients with HS and controls, respectively (Table 3).

The unadjusted risk of RA was numerically, but not statistically, higher in patients with HS relative to controls (HR, 1.75; 95\% CI, 0.99-3.90; $p=0.055$; Fig. 1). In a stratified analysis, the risk of RA was only elevated in individuals older than 30 years (HR, 1.94; 95\% CI, 1.02-3.69; $p=0.043$ ). The risk of RA in HS fell short of significance
Table 1 Descriptive characteristics of the study population

\begin{tabular}{|c|c|c|c|}
\hline Characteristic & $\begin{array}{l}\text { Patients with HS } \\
(\mathrm{N}=6779)\end{array}$ & Controls $(\mathrm{N}=33,260)$ & $p$ value \\
\hline \multicolumn{4}{|l|}{ Age, years } \\
\hline Mean (SD) & $33.1(15.1)$ & $33.1(15.1)$ & \multirow[t]{2}{*}{0.817} \\
\hline Median (range) & $30.0(0.6-88.7)$ & $30.0(0.6-88.8)$ & \\
\hline \multicolumn{4}{|l|}{ Sex, N (\%) } \\
\hline Male & $2708(39.9 \%)$ & $13,347(40.1 \%)$ & \multirow[t]{2}{*}{0.780} \\
\hline Female & $4071(60.1 \%)$ & $19,913(59.9 \%)$ & \\
\hline \multicolumn{4}{|l|}{ Ethnicity, N (\%) } \\
\hline Jews & $5607(82.7 \%)$ & $27,413(82.4 \%)$ & \multirow[t]{2}{*}{0.768} \\
\hline Arabs & $1172(17.3 \%)$ & $5846(17.6 \%)$ & \\
\hline \multicolumn{4}{|l|}{$\mathrm{BMI}, \mathrm{mg} / \mathrm{kg}^{2}$} \\
\hline Mean (SD) & $27.2(6.5)$ & $24.7(5.6)$ & $<0.001$ \\
\hline Smoking, N (\%) & $3591(53.0 \%)$ & $11,469(34.5 \%)$ & $<0.001$ \\
\hline Diabetes Mellitus, N (\%) & $698(10.3 \%)$ & $2236(6.7 \%)$ & $<0.001$ \\
\hline Hyperlipidemia, N (\%) & $1972(29.1 \%)$ & $7690(23.1 \%)$ & $<0.001$ \\
\hline Hypertension, N (\%) & $853(12.6 \%)$ & $3220(9.7 \%)$ & $<0.001$ \\
\hline \multicolumn{4}{|l|}{ Charlson comorbidity score } \\
\hline Mean score (SD) & $0.5(1.2)$ & $0.4(1.0)$ & $<0.001$ \\
\hline None $(0)$ & $4891(72.1 \%)$ & $26,375(79.3 \%)$ & $<0.001$ \\
\hline Moderate (1-2) & $1469(21.7 \%)$ & $5531(16.6 \%)$ & $<0.001$ \\
\hline Severe $(\geq 3)$ & $419(6.2 \%)$ & $1355(4.1 \%)$ & $<0.001$ \\
\hline
\end{tabular}

$H S$ hidradenitis suppurativa, $N$ number, $S D$ standard deviation, $B M I$ body mass index 
Table 2 The odds of hidradenitis suppurativa in patients with a preexisting diagnosis of rheumatoid arthritis stratified by age, gender, ethnicity, and latency (case-control study design)

\begin{tabular}{|c|c|c|c|c|}
\hline Subgroup & $\begin{array}{l}\text { Preexisting RA in patients with } \\
\mathrm{HS} \mathrm{N}(\%)(\mathrm{N}=6763)^{*}\end{array}$ & $\begin{array}{l}\text { Preexisting RA in controls } \\
\mathrm{N}(\%)(\mathrm{N}=33,215)^{*}\end{array}$ & OR $(95 \% \mathrm{CI})$ & $\begin{array}{l}\text { Univariate } \\
p \text { value }\end{array}$ \\
\hline All & $33(0.5 \%)$ & $102(0.3 \%)$ & $1.59(1.07-2.36)$ & 0.019 \\
\hline \multicolumn{5}{|l|}{ Age, years } \\
\hline$<20$ & $4(0.3 \%)$ & $7(0.1 \%)$ & $2.80(0.82-9.59)$ & 0.086 \\
\hline $20-40$ & $6(0.2 \%)$ & $29(0.2 \%)$ & $1.01(0.42-2.44)$ & 0.976 \\
\hline$\geq 40$ & $23(1.3 \%)$ & $66(0.7 \%)$ & $1.73(1.08-2.79)$ & 0.022 \\
\hline \multicolumn{5}{|l|}{ Gender } \\
\hline Male & $9(0.3 \%)$ & $20(0.1 \%)$ & $2.22(1.01-4.89)$ & 0.041 \\
\hline Female & $117(2.9 \%)$ & $438(2.2 \%)$ & $1.44(0.91-2.27)$ & 0.118 \\
\hline \multicolumn{5}{|l|}{ Ethnicity } \\
\hline Jews & $26(0.5 \%)$ & $90(0.3 \%)$ & $1.42(0.91-2.19)$ & 0.118 \\
\hline Arabs & $7(0.6 \%)$ & $12(0.2 \%)$ & $2.93(1.15-7.45)$ & 0.018 \\
\hline \multicolumn{5}{|c|}{ Latency after the diagnosis of RA } \\
\hline $0-5$ years & $7(0.1 \%)$ & $29(0.1 \%)$ & $1.19(0.52-2.71)$ & 0.686 \\
\hline $6-10$ years & $11(0.2 \%)$ & $36(0.1 \%)$ & $1.50(0.76-2.95)$ & 0.235 \\
\hline$>10$ years & $15(0.2 \%)$ & $37(0.1 \%)$ & $1.99(1.09-3.63)$ & 0.022 \\
\hline \multicolumn{5}{|l|}{ Multivariate analyses } \\
\hline Age- and sex-adjusted OR & & & $1.61(1.08-2.38)$ & 0.019 \\
\hline Fully-adjusted OR** & & & $1.66(1.11-2.49)$ & 0.014 \\
\hline
\end{tabular}

*Excluding patients with a diagnosis of RA after HS or recruitment

**Adjusting for age, sex, ethnicity, BMI, and comorbidities

$H S$ hidradenitis suppurativa, $R A$ rheumatoid arthritis, $O R$ odds ratio, $N$ number, $C I$ confidence interval

Bold significant value

Table 3 Incidence rates and hazard ratio of new-onset rheumatoid arthritis among patients with hidradenitis suppurativa (cohort study design)

\begin{tabular}{|c|c|c|}
\hline & $\mathrm{HS}(\mathrm{N}=6746)^{*}$ & Control $(\mathrm{N}=33,158)^{*}$ \\
\hline Follow-up time, PY & $37,591.7$ & $184,763.3$ \\
\hline Median follow-up time, years (range) & $6.1(0.1-18.7)$ & $6.1(0.1-18.7)$ \\
\hline Number of new-onset RA events & 16 & 45 \\
\hline Incidence rate / 10,000 PY (95\% CI) & $4.3(2.5-6.8)$ & $2.4(1.8-3.2)$ \\
\hline Unadjusted HR (95\% CI) [ $p$ value $]$ & $1.75(0.99-3.90)[0.055]$ & Ref. \\
\hline Male-specific HR (95\% CI) [ $p$ value] & $1.79(0.57-5.62)[0.319]$ & Ref. \\
\hline Female-specific HR (95\% CI) [ $p$ value] & $1.73(0.90-3.34)[0.102]$ & Ref. \\
\hline$<30$ years-specific HR $(95 \% \mathrm{CI})\left[p\right.$ value $^{\mathrm{a}}$ & $1.23(0.35-4.34)[0.753]$ & Ref. \\
\hline$\geq 30$ years-specific HR $(95 \%$ CI $)\left[p\right.$ value $^{\mathrm{a}}$ & $1.94(1.02-3.69)[0.043]$ & Ref. \\
\hline Jews-specific HR (95\% CI) [ $p$ value] & $1.50(0.79-2.87)[0.216]$ & Ref. \\
\hline Arabs-specific HR (95\% CI) [ $p$ value] & $3.36(0.95-11.91)[0.061]$ & Ref. \\
\hline Age- and sex-Adjusted HR (95\% CI) [ $p$ value] & $1.75(0.99-3.09)[0.055]$ & Ref. \\
\hline Fully adjusted HR (95\% CI) $[p \text { value }]^{\mathrm{b}}$ & $1.45(0.77-2.72)[0.249]^{\mathrm{a}}$ & Ref. \\
\hline
\end{tabular}

$H S$ hidradenitis suppurativa, $R A$ rheumatoid arthritis, $H R$ hazard ratio, $C I$ confidence interval, $P Y$ personyear

*Excluding patients with a diagnosis of RA before HS or recruitment

${ }^{\text {a }}$ Cutoff age was set at 30 since it represents the median age of study participants

${ }^{\mathrm{b}}$ Adjusted for age, sex, ethnicity, BMI, and comorbidities (per modified CCI)

Bold significant value. 
Fig. 1 Kaplan Meier curves demonstrating the cumulative incidence of RA among patients with HS and controls

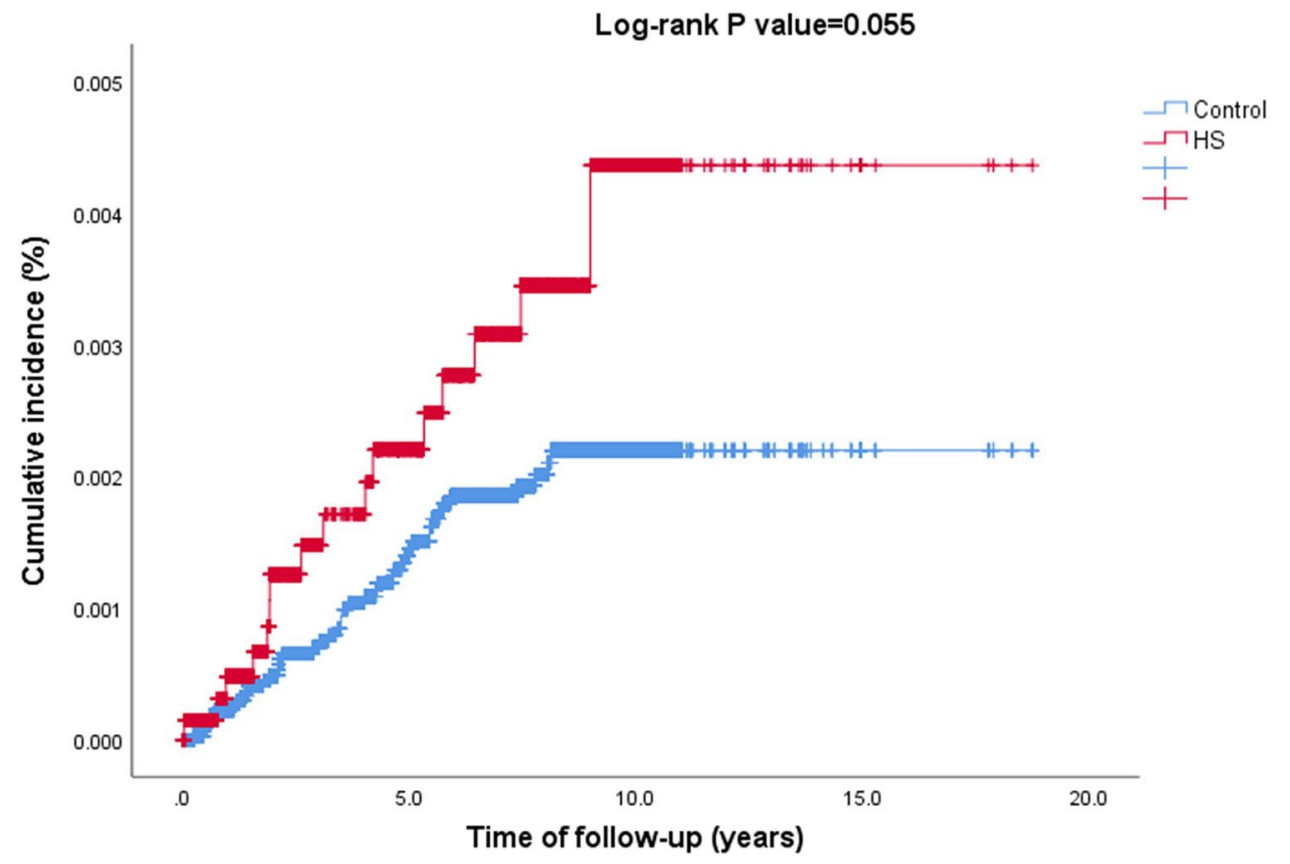

after adjusting for age and sex (age- and sex-adjusted HR, $1.75 ; 95 \%$ CI, 0.99-3.09; $p=0.055)$ as well as for age, sex, ethnicity, BMI, and comorbidities (fully-adjusted HR, 1.45; 95\% CI, 0.77-2.72; $p=0.249$; Table 3).

\section{Features of patients with hidradenitis suppurativa and comorbid rheumatoid arthritis}

The last endpoint of the study was to assess the characteristics of patients with HS and comorbid RA relative to the remaining patients with HS. Patients with HS and comorbid RA were significantly older at the onset of HS, had greater CCI scores, and higher lifetime prevalence of diabetes mellitus, hypertension, and hyperlipidemia (Table 4).

We then evaluated all-cause mortality of patients with HS and comorbid RA as compared to the remaining patients with HS. The risk of all-cause mortality was comparable between the two subgroups (HR, 2.59; $95 \%$ CI, 0.64-10.47; $p=0.183$; Supplementary figure).

Table 4 Characteristics of patients with hidradenitis suppurativa and comorbid rheumatoid arthritis relative to the remaining patients with hidradenitis suppurativa

\begin{tabular}{|c|c|c|c|c|c|}
\hline & $\begin{array}{l}\text { Coexistent HS and } \\
\text { RA }(n=49)\end{array}$ & $\begin{array}{l}\text { HS without RA } \\
(n=6730)\end{array}$ & OR & $95 \% \mathrm{CI}$ & $p$ value \\
\hline Age at the onset of HS, years; mean (SD) & $48.5(18.1)$ & $33.0(15.0)$ & $1.70^{\mathrm{a}}$ & $1.46-1.98$ & $<0.001$ \\
\hline Female sex, $n(\%)$ & $36(73.5 \%)$ & $4035(60.0 \%)$ & 1.85 & $0.98-3.50$ & 0.054 \\
\hline Jewish ethnicity, $n(\%)$ & $38(77.6 \%)$ & $5569(82.7 \%)$ & 0.72 & $0.37-1.41$ & 0.338 \\
\hline Body mass index; $\mathrm{Kg} / \mathrm{m}^{2}$, mean (SD) & $34.7(23.8)$ & $27.5(20.5)$ & $1.00^{\mathrm{b}}$ & $0.99-1.01$ & 0.116 \\
\hline Modified Charlson Comorbidity index; mean (SD) & $1.3(2.0)$ & $0.5(1.1)$ & $1.35^{\mathrm{c}}$ & $1.18-1.54$ & $<0.001$ \\
\hline Diabetes mellitus, $n(\%)$ & $18(36.7 \%)$ & $680(10.1 \%)$ & 5.17 & $2.88-9.28$ & $<0.001$ \\
\hline Hypertension, $n(\%)$ & $18(36.7 \%)$ & $835(12.4 \%)$ & 4.10 & $2.28-7.36$ & $<0.001$ \\
\hline Hyperlipidemia, $n(\%)$ & $31(63.3 \%)$ & $1941(28.8 \%)$ & 4.25 & $2.37-7.61$ & $<0.001$ \\
\hline Smoking, $n(\%)$ & $26(53.1 \%)$ & $3565(96.9 \%)$ & 1.00 & $0.57-1.76$ & 0.990 \\
\hline
\end{tabular}

Change in OR per 10 years ${ }^{\mathrm{a}}, \mathrm{mg} / \mathrm{kg}^{2}$ unit $^{\mathrm{b}}$ or point in CCI score ${ }^{\mathrm{c}}$.

$H S$ hidradenitis suppurativa, $R A$ rheumatoid arthritis, $O R$ odds ratio, $C I$ confidence interval, $P Y$ person-year, $S D$ standard deviation 


\section{Discussion}

The current large-scale population-based study revealed that a history of RA confers a 1.6-times increased risk of developing HS. Relative to other patients with HS, those with HS and comorbid RA were significantly older at the onset of HS, had a greater burden of comorbidities, and higher lifetime prevalence of metabolic syndrome.

\section{The current literature in comparison to our findings}

Patients with RA may experience a wide array of cutaneous manifestations $[14,15]$. These include, among others, rheumatoid nodules, accelerated rheumatoid nodulosis, rheumatoid vasculitis, palisaded neutrophilic and granulomatosis dermatitis, Felty syndrome, and pyoderma gangrenosum $[14,15]$. HS, on the other hand, has been found to coexist with several musculoskeletal diseases, mainly spondyloarthropathies $[16,17]$. In their retrospective cohort study following 640 patients with HS, Richette et al. [18] estimated the prevalence of ankylosing spondylitis at $3.7 \%$ based on the ESSG criteria, whereas none of their patients was diagnosed with RA.

While the coexistence of HS with RA has been reported anecdotally [7, 8], our knowledge about the association between these conditions is sparse. In a recent populationbased study from South Korea, the distribution of comorbidities among patients with HS has been systematically investigated [19]. An increased lifetime prevalence of RA among patients with HS has emerged, yielding a $30 \%$ increase in the OR $(1.31 ; 95 \% \mathrm{CI}, 1.16-1.48)$ relative to control individuals [19]. While the aforementioned study enrolled a larger sample size, its analysis was hampered by a cross-sectional design which did not enable to detect the temporal relationship between the diagnosis of HS and RA. Our study, contrariwise, estimated the bidirectional association of HS by employing two different study designs.

A recent retrospective cohort study followed patients with HS to estimate the risk of developing four types of inflammatory arthritis: ankylosing spondylitis, psoriatic arthritis, other spondyloarthritis, and RA [20]. Following propensity score matching, the risk of RA was significantly increased among patients with HS (HR, 1.16; 95\% CI, 1.03-1.31). Given its unidirectional nature, this study was underpowered to investigate the odds of HS after RA [20]. Our study demonstrated a prominent trend towards susceptibility to RA in patients with HS and a significantly increased risk of RA among HS patients older than 30 years of age. Therefore, our findings lend weight, at least to a certain extent, to the aforementioned study [20].
One may assume that this association might have reached the level of statistical significance if a larger sample size was utilized. Further population-based research is warranted to reproduce these findings. Our study denotes that the association of HS with RA was stronger among males. This finding accords with that reported by Lee et al. [19] in their Korean cohort.

\section{Putative interpretations of the observed association}

The pathomechanism underlying the association of HS with RA is yet to be delineated. However, both diseases share some common pathogenic themes. The coexistence of HS and RA was postulated to occur through the exposure of cutaneous antigens that might lead to the deposition of immune complexes in the synovial fluid [21]. Although the final common pathway is different, there are commonalities in the interplay of $\mathrm{T}$ and $\mathrm{B}$ cell and the activation of proinflammatory cytokines in both diseases, including the overproduction of tumor necrosis factor (TNF) as well as other essential proinflammatory mediators such as interleukin (IL)-6 and IL-1. Therefore, both conditions exhibit a satisfactory clinical response to TNF antagonists [22, 23]. Recent evidence suggested that systemic complement activation might exert a pathogenic role in both HS [24, 25] and RA [26]. Epidemiologically, both diseases are more prevalent in females and cigarette smokers, signifying a predisposing role played by estrogen and nicotine, respectively [27, 28].

\section{Strengths and limitations}

To the best of our knowledge, the current paper represents the first population-based study aiming to evaluate the bidirectional association between HS and RA. The large sample size provides the study with sufficient power to exclude chance as the basis for the findings. Owing to retrieving clinical data from all tiers of healthcare facilities, the study is less susceptible to selection bias than other study designs. The utilization of two study designs enables evaluation of the bidirectional association between the conditions of interests and provides a thorough insight into the temporal relationship between the diseases. Although RA lies in the differential diagnosis of spondyloarthropathies, misclassification in the current study is highly unlikely since the diagnosis of RA relied on documentation by board-certified rheumatologists. Confining cases of HS to those coded by certified dermatologists substantiates the validity of the diagnosis since HS is a clinical diagnosis that should be not mistaken by a trained dermatologist. The current study is not devoid of limitations. Lack of data regarding the clinical characteristics, severity, and immunoserological profile (like seropositivity of anti-CCP and RF antibodies) of patients was the main of these drawbacks. 
In conclusion, the current population-based study attested that a history of RA is associated with an elevated risk of subsequent HS. In addition, HS patients older than 30 years are at an increased risk of developing RA. Compared to other patients with HS, those with HS and comorbid RA were significantly older, had a greater burden of comorbidities, and higher lifetime prevalence of metabolic syndrome. Overall survival was comparable between the two subgroups. Our findings emphasize the significance of a multidisciplinary approach as clinicians managing patients with both diseases should be aware of symptoms suggestive of HS and RA and refer patients to specialists at an early stage. In patients with a dual diagnosis of HS and RA, treatment modalities that have been proved effective for both conditions might be preferred. Further research is necessary to delineate the pathomechanism underlying this observation.

Supplementary Information The online version contains supplementary material available at https://doi.org/10.1007/s12026-021-09221-4.

\section{Acknowledgements None}

Funding Open Access funding enabled and organized by Projekt DEAL. None.

\section{Declarations}

Conflict of interest ADC served as an advisor, investigator, or speaker for Abbvie, BI, Dexcel Pharma, Janssen, Novartis, Perrigo, Pfizer, and Rafa. None of the other authors have any conflicts of interest to declare.

Open Access This article is licensed under a Creative Commons Attribution 4.0 International License, which permits use, sharing, adaptation, distribution and reproduction in any medium or format, as long as you give appropriate credit to the original author(s) and the source, provide a link to the Creative Commons licence, and indicate if changes were made. The images or other third party material in this article are included in the article's Creative Commons licence, unless indicated otherwise in a credit line to the material. If material is not included in the article's Creative Commons licence and your intended use is not permitted by statutory regulation or exceeds the permitted use, you will need to obtain permission directly from the copyright holder. To view a copy of this licence, visit http://creativecommons.org/licenses/by/4.0/.

\section{References}

1. Saunte DML, Jemec GBE. Hidradenitis suppurativa: Advances in diagnosis and treatment. JAMA - J Am Med Assoc. 2017. p. 2019-32.

2. Goldburg SR, Strober BE, Payette MJ. Hidradenitis suppurativa: Epidemiology, clinical presentation, and pathogenesis. J Am Acad Dermatol Mosby Inc.; 2020. 82: 1045-58.

3. Jemec GBE, Kimball AB. Hidradenitis suppurativa: Epidemiology and scope of the problem. J. Am. Acad. Dermatol. [Internet]. Mosby Inc.; 2015;73:S4-7.

4. Revuz JE, Canoui-Poitrine F, Wolkenstein P, Viallette C, Gabison G, Pouget F, et al. Prevalence and factors associated with hidradenitis suppurativa: Results from two case-control studies. J. Am. Acad. Dermatol. [Internet]. J Am Acad Dermatol; 2008 [cited 2021 May 23];59:596-601. Available from: https://pubmed. ncbi.nlm.nih.gov/18674845/.

5. Nguyen T V., Damiani G, Orenstein LAV, Hamzavi I, Jemec GB. Hidradenitis suppurativa: an update on epidemiology, phenotypes, diagnosis, pathogenesis, comorbidities and quality of life [Internet]. J. Eur. Acad. Dermatology Venereol. Blackwell Publishing Ltd; 2021 [cited 2021 May 23]. p. 50-61. Available from: https:// pubmed.ncbi.nlm.nih.gov/32460374/.

6. Smolen JS, Aletaha D, McInnes IB. Rheumatoid arthritis [Internet]. Lancet. Lancet Publishing Group; 2016. p. 2023-38.

7. Alam MS, LaBelle B. Treatment of lichen planopilaris with adalimumab in a patient with hidradenitis suppurativa and rheumatoid arthritis. JAAD Case Reports [Internet]. 2020;6:219-21.

8. Garcovich S, De Simone C, Genovese G, Berti E, Cugno M, Marzano AV. Paradoxical skin reactions to biologics in patients with rheumatologic disorders. Front Pharmacol. 2019. https:// doi.org/10.3389/fphar.2019.00282.

9. Kridin K, Jones VA, Patel PM, Gibson FT, Amber KT, Cohen AD. Hidradenitis suppurativa and pemphigus: a cross-sectional study. Arch Dermatol Res. 2020;312:501-5.

10. Kridin K, Amber KT, Comaneshter D, Cohen AD. Amyloidosis in hidradenitis suppurativa: a cross-sectional study and review of the literature. Clin Exp Dermatol. 2020;45:565-71.

11. Kridin K, Jones VA, Patel PM, Zelber-Sagi S, Hammers CM, Damiani G, et al. Patients with pemphigus are at an increased risk of developing rheumatoid arthritis: a large-scale cohort study. Immunol Res. 2020;68:373-8.

12. Kridin K, Damiani G, Cohen AD. Rheumatoid arthritis and pyoderma gangrenosum: a population-based case-control study. Clin Rheumatol. 2021;40:521-8.

13. Charlson ME, Pompei P, Ales KL, MacKenzie CR. A new method of classifying prognostic comorbidity in longitudinal studies: development and validation. J Chronic Dis. 1987;40:373-83.

14. Lora V, Cerroni L, Cota C. Skin manifestations of rheumatoid arthritis. G Ital di Dermatologia e Venereol. 2018;153:243-55.

15. Sayah A, English JC. Rheumatoid arthritis: A review of the cutaneous manifestations. J. Am. Acad. Dermatol. 2005. p. 191-209.

16. Rosner IA, Burg CG, Wisnieski JJ, Schacter BZ, Richter DE. The clinical spectrum of the arthropathy associated with hidradenitis suppurativa and acne conglobata. J Rheumatol. 1993;20:684-7.

17. Shlyankevich J, Chen AJ, Kim GE, Kimball AB (2014) Hidradenitis suppurativa is a systemic disease with substantial comorbidity burden: A chart-verified case-control analysis. J Am Acad Dermatol Mosby Inc 71:1144-50.

18. Richette P, Molto A, Viguier M, Dawidowicz K, Hayem G, Nassif A, et al. Hidradenitis suppurativa associated with spondyloarthritis - Results from a multicenter national prospective study. J Rheumatol. 2014;41:490-4.

19. Lee JH, Kwon HS, Jung HM, Kim GM, Bae JM. Prevalence and comorbidities associated with hidradenitis suppurativa in Korea: a nationwide population-based study. J Eur Acad Dermatology Venereol. 2018;32:1784-90.

20. Schneeweiss MC, Kim SC, Schneeweiss S, Rosmarin D, Merola JF. Risk of inflammatory arthritis after a new diagnosis of hidradenitis suppurativa. JAMA Dermatol. 2020;156:342-5.

21. Miller IM, McAndrew RJ, Hamzavi I. Prevalence, Risk Factors, and Comorbidities of Hidradenitis Suppurativa. Dermatol. Clin. 2016. p. 7-16.

22. Kimball AB, Okun MM, Williams DA, Gottlieb AB, Papp KA, Zouboulis CC, et al. Two Phase 3 Trials of Adalimumab for Hidradenitis Suppurativa. N Engl J Med. 2016;375:422-34.

23. Scott DL, Ibrahim F, Farewell V, Go'Keeffe A, Walker D, Kelly $\mathrm{C}$, et al. Tumour necrosis factor inhibitors versus combination 
intensive therapy with conventional disease modifying anti-rheumatic drugs in established rheumatoid arthritis: TACIT non-inferiority randomised controlled trial. BMJ [Internet]. BMJ Publishing Group; 2015;350.

24. Grand D, Navrazhina K, Frew JW. Integrating complement into the molecular pathogenesis of Hidradenitis Suppurativa. Exp. Dermatol. [Internet]. Blackwell Publishing Ltd; 2020;29:86-92.

25. Kanni T, Zenker O, Habel M, Riedemann N, Giamarellos-Bourboulis EJ. Complement activation in hidradenitis suppurativa: a new pathway of pathogenesis? Br. J. Dermatol. [Internet]. Blackwell Publishing Ltd; 2018;179:413-9.

26. Hashimoto M, Hirota K, Yoshitomi H, Maeda S, Teradaira S, Akizuki S, et al. Complement drives Th17 cell differentiation and triggers autoimmune arthritis. J. Exp. Med. [Internet]. J Exp Med; 2010;207:1135-43.
27. Ingram JR. The epidemiology of hidradenitis suppurativa*. Br. J. Dermatol. [Internet]. Blackwell Publishing Ltd; 2020;183:990-8.

28. Ishikawa Y, Terao C. The Impact of Cigarette Smoking on Risk of Rheumatoid Arthritis: A Narrative Review [Internet]. Cells. NLM (Medline); 2020 [cited 2021 May 22]. Available from: https:// pubmed.ncbi.nlm.nih.gov/32092988/.

Publisher's note Springer Nature remains neutral with regard to jurisdictional claims in published maps and institutional affiliations. 